\title{
Special issue on Nature-inspired systems for parallel, asynchronous and decentralised environments
}

\author{
Enda Ridge $^{\mathrm{a}}$, Edward Curry ${ }^{\mathrm{b}}$, Daniel Kudenko ${ }^{\mathrm{a}}$ and Dimitar Kazakov ${ }^{\mathrm{a}}$ \\ ${ }^{a}$ Department of Computer Science, The University of York, York YO10 5DD, UK \\ ${ }^{\mathrm{b}}$ Department of Information Technology, National University of Ireland, Galway, Ireland
}

Keywords: Nature-inspired systems, parallel, asynchronous, decentralised environments

\section{Introduction}

Nature-inspired algorithms such as genetic algorithms, particle swarm optimisation and ant colony algorithms are the state-of-the-art solution technique for some problems. Furthermore, their populationbased stochastic search approach promises desirable algorithm features such as anytime decentralised solution and robustness to problem change. However, the efficient pursuit of more accurate solutions leads researchers to appeal to centralised, highly tuned and sequential implementations that are only loosely related to their successful natural counterparts. This renders them brittle in the face of the dynamism of changing problem specifications and operating conditions and limits their usefulness to industry's direction of increasing distribution, decentralisation and adaptability.

Emerging computing environments such as autonomic computing, ubiquitous computing, Peer-to-Peer systems, the Grid and the Semantic Web demand the interaction of large numbers of decentralised, parallel, asynchronous, and distributed software entities in a standardised fashion. If nature-inspired algorithms are to make an impact on these emerging computing environments, disciplined scientific and engineering investigations must be undertaken into the successful transfer of these algorithms, techniques and infrastructures into such environments.

\section{Contributions}

This special issue comprises eight contributions.

The issue begins with a roadmap for research in the field [8]. Important directions for future research and current challenges are highlighted.

Optimising the quality of service for dynamic software compositions is challenging. The task is further complicated when performed within the parallel, asynchronous, and decentralised environments faced by web services. Cao et al. [2], present a solution to this problem using one of the most well-established natureinspired techniques, the genetic algorithm, to optimise the cost-driven selection of web services.

Grid environments have been a popular choice for the application of nature-inspired techniques. The placement of application services onto available nodes within a grid environment is a complex and difficult task. Musunoori and Horn [6] tackle this problem with the use of three algorithms based on the concepts of learning automata and ant systems. This achieves a decentralised multiagent approach for solving service partitioning within grid environments.

Researchers from Italy's Institute for High Performance Computing and Networking created an antbased approach for resource discovery and management within grids. This work [3] presents a decen- 
tralised and self-organising approach for building grid resource information systems.

In addition to applying nature-inspired techniques within emerging computing environments, much work has been done on the design methodology for natureinspired systems. In an approach inspired by Minsky's Society of Mind concept, the open-source AKIRA framework [4] is designed to build distributed, decentralised, parallel, and asynchronous systems using modular software architectures.

Another interesting use of the ant metaphor is the Personal Intelligent Agents Framework (PIAF) [4]. This framework, developed at the Université de Tours, France, provides an ant colony epidemic-based approach to selective information dissemination within Peer-to-Peer networks. PIAF builds an estimated interest profile based on the pheromone trails associated with connections between peers.

Jones and Saeed [5] demonstrate a parallel and decentralised ant-based approach to image processing. Their PixieDust multi-agent programming framework uses a colony of ants to process an image. The image provides stimuli to the ant, allowing emergent pattern formation to take place.

Researchers at the University of Le Havre developed a decentralised nature-inspired algorithm called $\mathrm{AntCO}^{2}$ [1] based on the principle of ant trailfollowing. Utilising this behaviour the algorithm can optimise the distribution of computation and minimise the communication costs in a distributed simulator.

\section{Acknowledgments}

We are grateful to the many reviewers without whom this special issue would not have been possible. We thank the authors and the presenters at the original NISPADE event at the AISB Convention in Bristol 2006 for their contributions and discussions that helped shape this special issue. We are also very grateful to the Multi-agent and Grid Systems journal editors, Rainer Unland and Huaglory Tianfield for the opportunity to edit this special issue and for their support throughout the process.

\section{References}

[1] C. Bertelle, A. Dutot, F. Guinand and D. Olivier, Organization Detection for Dynamic Load Balancing in Individual-Based Simulations, Multi-Agent and Grid Systems 3(1) (2007).

[2] C. Li, L. Minglu and C. Jian, Using Genetic Algorithm to Implement Cost-Driven Web Service Selection, Multi-Agent and Grid Systems 3(1) (2007).

[3] A. Forestiero, C. Mastroianni and G. Spezzano, A Decentralized Ant-Inspired Approach for Resource Management and Discovery in Grids, Multi-Agent and Grid Systems 3(1) (2007).

[4] C. Guáfret, N. Monmarché and M. Simane, A biology-inspired model for the automatic dissemination of information in P2P networks, Multi-Agent and Grid Systems 3(1) (2007).

[5] J. Jones and M. Saeed, Image Enhancement - An Emergent Pattern Formation Approach via Decentralised Multi-Agent Systems, Multi-Agent and Grid Systems 3(1) (2007).

[6] S.B. Musunoori and G. Horn, Application Service Placement in Stochastic Grid Environments Using Learning and Ant Based Methods, Multi-Agent and Grid Systems 3(1) (2007).

[7] G. Pezzulo and G. Calvi, Designing Modular Architectures in the Framework AKIRA, Multi-Agent and Grid Systems 3(1) (2007).

[8] E. Ridge and E. Curry, A Roadmap of Nature-Inspired Systems Research and Development, Multi-Agent and Grid Systems 3(1) (2007). 